\title{
On special $L$-values attached to metaplectic modular forms
}

\author{
Thanasis Bouganis ${ }^{1}$
}

Received: 1 December 2014 / Accepted: 6 March 2017 / Published online: 25 May 2017

(C) The Author(s) 2017. This article is an open access publication

\begin{abstract}
In this paper we establish some algebraic properties of special $L$-values attached to Siegel modular forms of half-integral weight, often called metaplectic modular forms. These results are motivated by some "exercises" left by Shimura to the reader in his marvellous book "Arithmeticity in the Theory of Automorphic Forms".
\end{abstract}

\section{Introduction}

This paper is the continuation of our earlier work [1], concerning special $L$-values attached to Siegel modular forms. In the previous work we considered Siegel modular forms of integral weight, and in this paper we consider the half-integral weight situation. As we mentioned in our previous paper, the origin of these two works is the work of Shimura on the algebraicity of these special $L$-values (both in integral and half-integral situation). Indeed, Shimura, in his admirable book "Arithmeticity in the Theory of Automorphic Forms" [10], establishes various algebraicity results of these special $L$-values. These results are established over an algebraic closure of $\mathbb{Q}$ (see for example Theorem 6.1 below), and Shimura leaves it as an "exercise" to the reader to establish more accurate results (i.e. exact field of definition and a Galois reciprocity law, see [10, p. 239, Remark 28.13]). We believe that it is important to have these results documented in the literature and this is exactly the goal of this and our previous work. Our main results are Theorems 6.2 and 6.4 below, which rely in turn to the definition of some particular automoprhic periods (see Theorem 5.2). The structure of this paper is very similar to the previous one [1], and for this reason we have shortened quite a lot of proofs which are similar to the previous work. However there are a few technical points that need to be settled differently than in the previous paper. We finally mention that similar questions can be considered for Hermitian modular forms, and we refer to our paper [2] for more on this.

Thanasis Bouganis

athanasios.bouganis@durham.ac.uk

1 Department of Mathematical Sciences, Durham University, South Rd., Durham DH1 3LE, UK 
Notation Our main reference is the book of Shimura [10], and hence we have adopted most of the notation used there. There is only a few notational differences, perhaps the one worth emphasizing, is that we use the $L$ notation for our functions ( $L$-functions) where in [10] the zeta notation, $Z$, is used.

\section{Siegel modular forms}

In this section we introduce the notion of a Siegel modular form of integral and half-integral weight both from a classical and adelic point of view. This section is very similar to the corresponding one in our previous paper [1]. We follow closely the book of Shimura [10, Chapter I].

For a positive integer $n \in \mathbb{N}$ we define the matrix $\eta_{n}:=\left(\begin{array}{cc}0 & -I_{n} \\ I_{n} & 0\end{array}\right)$ and for any commutative ring $A$ with an identity the group $S p_{n}(A):=\left\{\left.\alpha \in G L_{2 n}(A)\right|^{t} \alpha \eta_{n} \alpha=\eta_{n}\right\}$. The group $S p_{n}(\mathbb{R})$ acts on the Siegel upper half space $\mathbb{H}_{n}:=\left\{z \in M_{n}(\mathbb{C}) \mid{ }^{t} z=z, \operatorname{Im}(z)>0\right\}$,

$$
\alpha \cdot z:=\left(a_{\alpha} z+b_{\alpha}\right)\left(c_{\alpha} z+d_{\alpha}\right)^{-1}, \quad \alpha=\left(\begin{array}{cc}
a_{\alpha} & b_{\alpha} \\
c_{\alpha} & d_{\alpha}
\end{array}\right) \in S p_{n}(\mathbb{R})
$$

where $*_{\alpha}$ is an $n \times n$ matrix.

Let now $F$ be a totally real field of degree $d$, discriminant $D_{F}$ and we denote by $\mathcal{O}$ its ring of integers. We write $\infty$ for the set of archimedean places of $F, \mathbf{f}$ for the finite ones and we set $G:=S p_{n}(F)$. We write $G_{\mathbb{A}}$ for the adelic group and we decompose $G_{\mathbb{A}}=G_{\mathbf{f}} G_{\infty}$ where $G_{\infty}:=\prod_{v \in \infty} G_{v}$ and $G_{\mathbf{f}}:=\prod_{v \in \mathbf{f}}^{\prime} G_{v}$. For a fractional ideal $\mathfrak{b}$ and an integral idealc of $F$ we define the subgroup of $G_{\mathbb{A}}$,

$$
D\left[\mathfrak{b}^{-1}, \mathfrak{b} \mathfrak{c}\right]:=\left\{x \in G_{\mathbb{A}} \mid a_{x} \prec \mathcal{O}_{v}, b_{x} \prec \mathfrak{b}_{v}^{-1}, c_{x} \prec \mathfrak{b c}_{v}, d_{x} \prec \mathcal{O}_{v}, \forall v \in \mathbf{f}\right\},
$$

where we use the notation " $\prec$ " of Shimura, $x \prec I$ meaning that the $v$-component of $x$ is a matrix with entries in the fractional ideal $I$. Strong approximation for $G$ implies that $G_{\mathbb{A}}=G q D\left[\mathfrak{b}^{-1}, \mathfrak{b} \mathfrak{c}\right]$ for any $\mathfrak{b}, \mathfrak{c}$ and $q \in G_{\mathbf{f}}$. We define $\Gamma^{q}(\mathfrak{b}, \mathfrak{c}):=G \cap q D\left[\mathfrak{b}^{-1}, \mathfrak{b} \mathfrak{c}\right] q^{-1}$.

We denote by $M_{\mathbb{A}}$ the adelized metaplectic group, and write $p: M_{\mathbb{A}} \rightarrow G_{\mathbb{A}}$ for the canonical projection to $G_{\mathbb{A}}$. Further we write $C^{\theta}$ for the theta group defined for example in [10, Appendix A2.3] and $\Gamma^{\theta}=G \cap C^{\theta}$. We note that $D\left[\mathfrak{b}^{-1}, \mathfrak{b c}\right] \subset C^{\theta}$ if $\mathfrak{b}^{-1} \subset 2 \mathfrak{d}^{-1}$ and $\mathfrak{b c} \subset 2 \mathfrak{d}$.

For an element $\sigma \in \Gamma^{\theta}$ and $z \in \mathbb{H}$ we write $h_{\sigma}(z)$ for the $1 / 2$-weight factor of automorphy as defined for example in [10, Theorem 6.8]. For a $k \in \frac{1}{2} \mathbb{Z}$ we define the factor of automorphy

$$
j_{\sigma}(z)^{k}:=\left\{\begin{array}{l}
\operatorname{det}\left(c_{\sigma} z+d_{\sigma}\right)^{k}, \quad k \in \mathbb{Z}, \quad \sigma \in G, \\
h_{\sigma}(z) j_{\sigma}(z)^{[k]}, \quad k \notin \mathbb{Z}, \sigma \in \Gamma^{\theta} .
\end{array}\right.
$$

Given a Hecke character $\psi$ of $F$ of conductor dividing $\mathfrak{c}$ we define a character on $D\left[\mathfrak{b}^{-1}, \mathfrak{b c}\right]$ by $\psi(x)=\prod_{v \mid \mathfrak{c}} \psi_{v}\left(\operatorname{det}\left(d_{x}\right)_{v}\right)$ and a character, which we still denote by $\psi$, on $\Gamma^{q}$ by $\psi(\gamma):=\psi\left(q^{-1} \gamma q\right)$

We now write $\mathbb{Z}^{d}:=\prod_{v \in \infty} \mathbb{Z}$ and $\mathcal{H}:=\prod_{v \in \infty} \mathbb{H}_{n}$. Elements $k \in \frac{1}{2} \mathbb{Z}^{d}$ will be called weights, if $k \in \mathbb{Z}^{d}$ or $k=\left(k_{v}\right) \in \frac{1}{2} \mathbb{Z}^{d}$ and $k_{v} \in \mathbb{Z}+\frac{1}{2}$ for all $v$, in which case we say that $k$ is a half-integral weight. For a function $f: \mathcal{H} \rightarrow \mathbb{C}$ and a weight $k \in \mathbb{Z}^{d}$ (resp. $k \in \frac{1}{2} \mathbb{Z}^{d}$ and half-integral), we define,

$$
\left(\left.f\right|_{k} \alpha\right)(z):=j_{\alpha}(z)^{-k} f(\alpha z), \quad z \in \mathcal{H} .
$$


for $\alpha \in G$ (resp. $\alpha \in \Gamma^{\theta}$ ). Here we write $z=\left(z_{v}\right)_{v \in \infty}$ with $z_{v} \in \mathbb{H}_{n}$ and $\alpha \in G_{\infty}$ and define $j_{\alpha}(z)^{-k}:=\prod_{v} j_{\alpha_{v}}\left(z_{v}\right)^{k_{v}}$.

Let now $\Gamma$ be group of the form $\Gamma^{q}, q \in G_{\mathbf{f}}$ as above and $\psi$ a Hecke character. Moreover we fix a weight $k \in \frac{1}{2} \mathbb{Z}^{d}$, and in the half-integral case we will be also assuming that $\Gamma \subseteq \Gamma^{\theta}$. Then we define,

Definition 2.1 A function $f: \mathcal{H} \rightarrow \mathbb{C}$ is called a Siegel modular form of weight $k$ (or often called a metaplectic modular form if $k$ is half-integral) for the congruence subgroup $\Gamma$ and Nebentypus $\psi$ if

(1) $f$ is holomorphic,

(2) $\left.f\right|_{k} \gamma=\psi(\gamma) f$ for all $\gamma \in \Gamma$,

(3) $f$ is holomorphic at cusps.

The last condition is needed only if $F=\mathbb{Q}$ and $n=1$, which is then the usual condition of elliptic modular forms being holomorphic at cusps. The above defined space we will denote it by $\mathcal{M}_{k}(\Gamma, \psi)$.

For any $\gamma \in G$, we define $p_{\gamma}(z)=1$ if $k \in \mathbb{Z}^{d}$ and $p_{\gamma}(z)$ to be any branch of the square root of $j_{\gamma}(z)^{u}$ if $k$ is half-integral, where we write $u=(1, \ldots, 1) \in \mathbb{Z}^{d}$. Then we have a Fourier expansion of the form (see [10, pp. 33 and 73])

$$
p_{\gamma}(z)^{-1}\left(\left.f\right|_{[k]} \gamma\right)(z)=\sum_{s \in \mathfrak{S}} c(s, \gamma ; f) e_{\infty}(s z),
$$

where $\mathfrak{S}$ a lattice in $S_{+}:=\left\{s \in M_{n}(F) \mid s={ }^{t} s, s_{v} \geq 0, \forall v \in \infty\right\}$, and $e_{\infty}(x)=$ $\exp \left(2 \pi i \sum_{v} \operatorname{tr}\left(x_{v}\right)\right)$. An $f$ is called a cusp form if $c(s, \gamma ; f)=0$ for any $\gamma \in G$ and $s$ with $\operatorname{det}(s)=0$. The space of cusp forms we will be denoted by $\mathcal{S}_{k}(\Gamma, \psi)$. For a subfield $L$ of $\mathbb{C}$ we will be writing $\mathcal{M}_{k}(\Gamma, \psi, L)$ (resp. $\mathcal{S}_{k}(\Gamma, \psi, L)$ for the subspace of $\mathcal{M}_{k}(\Gamma, \psi)$ (resp. $\mathcal{S}_{k}(\Gamma, \psi)$ ) whose Fourier expansion at infinity (i.e. $\gamma=1$ above) has coefficients in $L$. Finally we note that for an element $\sigma \in \operatorname{Gal}(\overline{\mathbb{Q}} / \mathbb{Q}$ ) we may define (see [10, pp. 35 and 73] an action on $\mathcal{M}_{k}(\Gamma, \psi, \overline{\mathbb{Q}})$ by acting on the Fourier coefficients of the expansion at infinity.

We now turn to the adelic Siegel modular forms. We refer the reader to [9, Chapter II] and [10, pp. 166-167] for details. For a weight $k \in \frac{1}{2} \mathbb{Z}^{d}$, we set $\mathcal{G}_{\mathbb{A}}=G_{\mathbb{A}}$, and $p=1$ if $k$ is integral and $\mathcal{G}_{\mathbb{A}}=M_{\mathbb{A}}$, and $p$ as above, if $k$ is half-integral. Then

Definition 2.2 ([10, p. 166]) A function $\mathbf{f}: \mathcal{G}_{\mathbb{A}} \rightarrow \mathbb{C}$ is called adelic Siegel modular form of weight $k$ if

(1) $\mathbf{f}(\alpha x w)=\psi(w) j_{w_{\infty}}^{k}(\mathbf{i}) \mathbf{f}(x)$ for $\alpha \in G, w \in p^{-1}(D)$ with $w_{\infty}(\mathbf{i})=\mathbf{i}$,

(2) For every $p \in \mathcal{G}_{\mathbf{f}}$ there exists $f_{p} \in M_{k}\left(\Gamma^{p}, \psi\right)$, where $\Gamma^{p}:=G \cap p D p^{-1}$, such that $\mathbf{f}(p y)=\left(\left.f_{p}\right|_{k} y\right)(\mathbf{i})$ for every $y \in \mathcal{G}_{\infty}$.

Here $\mathbf{i}:=\left(i I_{n}, \ldots, i I_{n}\right) \in \mathcal{H}$. We write $\mathcal{M}_{k}(D, \psi)$ for this space. Strong approximation theorem for $S p_{n}$ gives $\mathcal{M}_{k}(D, \psi) \cong M_{k}\left(\Gamma^{q}, \psi\right)$ for any $q \in G_{\mathbf{f}}$, where in the half-integral case we assume that $\Gamma^{q} \subset \Gamma^{\theta}$. We define the space of automorphic cusp form $\mathcal{S}_{k}(D, \psi)$ to be the subspace of $\mathcal{M}_{k}(D, \psi)$ that is in bijection with $S_{k}\left(\Gamma^{q}, \psi\right)$ for any $q \in G_{\mathbf{f}}$ in the above bijection. Using the above bijection we also define $\mathcal{M}_{k}(D, \psi, L)$ for any subfield $L$ of $\mathbb{C}$ and an action of $\operatorname{Gal}(\overline{\mathbb{Q}} / \mathbb{Q})$ on $\mathcal{M}_{k}(D, \psi, \overline{\mathbb{Q}})$.

Finally for $q \in G L_{n}(F)_{\mathbb{A}}$ and $s \in S_{+\mathbb{A}}$ we set $x_{q, s}:=\left(\begin{array}{cc}q & s^{t} q^{-1} \\ 0 & { }^{t} q^{-1}\end{array}\right)$ and obtain an adelic $q$-expansion (see [10, pp. 167-168]),

$$
\mathbf{f}\left(x_{q, s}\right)=\operatorname{det}\left(q_{\infty}\right)^{[k]}|\operatorname{det}(q)|_{\infty}^{k-[k]} \sum_{\tau \in S_{+}} c(\tau, q ; \mathbf{f}) e_{\infty}\left(\mathbf{i}^{t} q \tau q\right) e_{\mathbb{A}}(\tau s),
$$


where for the definition of $e_{\mathbb{A}}$ to [10, p. 127], and in the case of half-integral we view $x_{q, s}$ in $M_{\mathbb{A}}$ using the canonical lift $P_{\mathbb{A}} \hookrightarrow M_{\mathbb{A}}$ of the adelic Siegel parabolic $P_{\mathbb{A}}$. Finally, in the half-integral weight situation, we set $\mu(\tau, q ; \mathbf{f}):=|\operatorname{det}(q)|_{F}^{1 / 2} c(\tau, q ; \mathbf{f})$.

\section{A reciprocity law on Eisenstein series}

In this section we recall a theorem proved in [1] concerning the action of the absolute Galois group on some Siegel type Eisenstein series. We start by fixing some notation. Let $k \in \frac{1}{2} \mathbb{Z}^{d}$ be a weight and let $\mathfrak{b}$ be a fractional ideal and $\mathfrak{c}$ an integral ideal of $F$. Further we consider a Hecke character $\chi$ of $F$ with infinity type $\chi_{\infty}(x)=x_{\infty}^{[k]}\left|x_{\infty}\right|^{[k]}$ and of conductor dividing $\mathfrak{c}$. Moreover in case that $k$ is half-integral we assume that $\mathfrak{b}$ and $\mathfrak{c}$ are such that $D\left[\mathfrak{b}^{-1}, \mathfrak{b} \mathfrak{c}\right] \subset D\left[2 \mathfrak{d}^{-1}, 2 \mathfrak{d}\right]$.

We write $E_{\mathbb{A}}(x, s):=E_{\mathbb{A}}(x, s ; \chi, \mathfrak{c}), x \in \mathcal{G}_{\mathbb{A}}, s \in \mathbb{C}$, and $E_{\mathbb{A}}^{*}(x, s)$ for the two adelic Eisenstein series of Siegel type defined as in [10, pp. 131-132]. Moreover we define,

$$
D_{\mathbb{A}}(x, s)=E_{\mathbb{A}}^{*}(x, s) \times\left\{\begin{array}{ll}
L_{\mathfrak{c}}(2 s, \chi) \prod_{i=1}^{[n / 2]} L_{\mathfrak{c}}\left(4 s-2 i, \chi^{2}\right), & k \in \mathbb{Z}^{d} ; \\
\prod_{i=1}^{[(n+1) / 2]} L_{\mathfrak{c}}\left(4 s-2 i-1, \chi^{2}\right), & k \notin \mathbb{Z}^{d} ;
\end{array},\right.
$$

and write $D(z, s ; k, \chi, \mathfrak{c})$ for the corresponding to $D_{\mathbb{A}}(x, s)$ classical Siegel modular form. For a number field $W$ we follow the notation in [10] and write $\mathcal{N}_{k}^{r}(W)$ for the space of $W$-rational nearly holomorphic forms of weight $k$ (for the meaning of $r$ we refer to [10]). The theorem below is due to Shimura [10, Theorem 17.9].

Theorem 3.1 (Shimura) Let $\Phi$ be the Galois closure of $F$ over $\mathbb{Q}$ and let $k \in \frac{1}{2} \mathbb{Z}^{d}$ be a weight with $k_{v} \geq(n+1) / 2$ for all $v \in \infty$ and $k_{v}-k_{v^{\prime}} \in 2 \mathbb{Z}$ for every $v, v^{\prime} \in \infty$. Let $\mu \in \frac{1}{2} \mathbb{Z}$ with $n+1-k_{v} \leq \mu \leq k_{v}$ and $\left|\mu-\frac{n+1}{2}\right|+\frac{n+1}{2}-k_{v} \in 2 \mathbb{Z}$ for all $v \in \infty$. Exclude the cases

(1) $\mu=(n+2) / 2, F=\mathbb{Q}$ and $\chi^{2}=1$,

(2) $\mu=0, \mathfrak{c}=\mathcal{O}$ and $\chi=1$,

(3) $0<\mu \leq n / 2, \mathfrak{c}=\mathcal{O}$ and $\chi^{2}=1$.

Then $D(z, \mu / 2 ; k, \chi, \mathfrak{c})$ belongs to $\pi^{\beta} \mathcal{N}_{k}^{r}\left(\Phi \mathbb{Q}_{a b}\right)$, where $r=(n / 2)(k-|\mu-(n+1) / 2| u-$ $\left.\frac{n+1}{2} u\right)$ except in the case where $n=1, \mu=2, F=\mathbb{Q}, \chi=1$ and $n>1, \mu=(n+3) / 2$, $F=\mathbb{Q}, \chi^{2}=1$. In these two case we have $r=n(k-\mu+2) / 2$. Moreover we have that $\beta=(n / 2) \sum_{v \in \infty}\left(k_{v}+\mu\right)-$ de where

$$
e= \begin{cases}{\left[(n+1)^{2} / 4\right]-\mu,} & \text { if } 2 \mu+n \in 2 \mathbb{Z} \text { and } \mu \geq \lambda ; \\ {\left[n^{2} / 4\right],} & \text { otherwise. }\end{cases}
$$

In [1, Theorem 3] we have proved the following reciprocity laws of the action of the Galois group $\operatorname{Gal}(\overline{\mathbb{Q}} / \Phi)$ on Eisenstein series, extending previous results of Feit [4]. Below $\mathbf{g}(\cdot)$ is a Gauss sum (see [1] for the normalization), and $C$ is a fixed primitive 8 th root of unity.

Theorem 3.2 Let $k \in \frac{1}{2} \mathbb{Z}^{d}$ with $k_{v} \geq(n+1) / 2$ for every $v \in \infty$. Let $\mu \in \frac{1}{2} \mathbb{Z}$ such that $n+1-k_{v} \leq \mu \leq k_{v}$ and $|\mu-(n+1) / 2|+(n+1) / 2-k_{v} \in 2 \mathbb{Z}$ for all $v \in \infty$. Then with a $\beta \in \mathbb{N}$ as in Theorem 3.1 we have

$$
\pi^{-\beta} D(z, \mu / 2 ; k, \chi, \mathfrak{c}) \in \mathcal{N}_{k}^{r}\left(\Phi \mathbb{Q}_{a b}\right),
$$


and for every $\sigma \in \operatorname{Gal}\left(\Phi \mathbb{Q}_{a b} / \Phi\right)$ we have

$$
\left(\frac{\pi^{-\beta} D(z, \mu / 2 ; k, \chi, \mathfrak{c})}{\omega(\chi)}\right)^{\sigma}=\frac{\pi^{-\beta} D\left(z, \mu / 2 ; k, \chi^{\sigma}, \mathfrak{c}\right)}{\omega\left(\chi^{\sigma}\right)},
$$

where $\omega(\chi)$ is given as follows:

(1) if $k \in \mathbb{Z}^{d}, \mu \geq(n+1) / 2$ :

$$
\omega(\chi)=i^{n|p|} \mathbf{g}(\chi) i^{\mu d+2 \mu[n / 2]-[n / 2]([n / 2]+1) d} D_{F}^{-b(n)} \mathbf{g}\left(\chi^{2[n / 2]}\right),
$$

where $p:=\frac{(k-\mu u)}{2}$ and $b(n)=0$ if $[n / 2]$ odd and $1 / 2$ otherwise.

(2) if $k \in \mathbb{Z}^{d}, \mu<(n+1) / 2$ :

$$
\omega(\chi)=i^{n|p|} \mathbf{g}(\chi)^{n} i^{-d n v} D_{F}^{-n \mu+3 n(n+1) / 4},
$$

where $v:=n+1-\mu$ and $p:=\frac{k-v u}{2}$.

(3) if $k \notin \mathbb{Z}^{d}$ and $\mu \geq(n+1) / 2$ :

(a) if $n$ is even

$$
\omega(\chi)=i^{n|p|} \mathbf{g}(\chi \phi)^{n} i^{-d n k} C D_{F}^{n k / 2+3 n(n+1) / 4},
$$

(b) if $n$ is odd

$$
\omega(\chi)=i^{n|p|} \mathbf{g}(\chi \phi)^{n} i^{-d n k} C\left|D_{F}\right|^{n \mu / 2+3 n(n+1) / 4} \mathbf{g}(\chi) D_{F}^{1 / 2}(2 i)^{-(\mu-n) d} b([n / 2]),
$$

where $p:=\frac{(k-\mu u)}{2}$ and $b(m)=i^{d}$ if $m$ is $m$ is odd and 1 otherwise and

(4) if $k \notin \mathbb{Z}^{d}$ and $\mu<(n+1) / 2$ :

$$
\omega(\chi)=i^{n|p|} \mathbf{g}(\chi \phi)^{n} i^{-d n v} C D_{F}^{-n(n+1-v)+3 n(n+1) / 4},
$$

where $v:=n+1-\mu$ and $p:=\frac{k-v u}{2}$.

The character $\phi$ in (iii) and (iv) above is induced by the character on $\Gamma^{\theta}$ defined as $h_{\gamma}(z)^{2}=$ $\phi(\gamma) j_{\gamma}(z)^{u}$ (see also the few lines in the proof of [10, Theorem 10.7]). In particular we have that

$$
\frac{\pi^{-\beta} D(z, \mu / 2 ; k, \chi, \mathfrak{c})}{\omega(\chi)} \in \mathcal{N}_{k}^{r}(\Phi(\chi)),
$$

where $\Phi(\chi)$ is the finite extension of $\Phi$ obtained by adjoining the values of the character $\chi$.

\section{The $L$-function attached to a metaplectic modular form and the Rankin-Selberg method}

In this section we collect various results of Shimura on the $L$-functions associated to halfintegral weight Siegel modular forms and the Rankin-Selberg method. Everything in this section is from the works of Shimura $[7,8,10]$. For notation not introduced in this section we refer to [10].

From now on, until the end of the paper, $k$ will always denote a half-integral weight. We fix a fractional ideal $\mathfrak{b}$ and an integral ideal $\mathfrak{c}$. We write $C=D\left[\mathfrak{b}^{-1}, \mathfrak{b} \mathfrak{c}\right]$, with the usual assumption on $\mathfrak{b}$ and $\mathfrak{c}$. For an integral ideal $\mathfrak{a}$ we write $T(\mathfrak{a})$ for the Hecke operator acting on half-integral Siegel modular forms as defined by Shimura in [10, p. 175] or [7]. 
We consider an $0 \neq \mathbf{f} \in \mathcal{S}_{k}(C, \psi)$ with $\mathbf{f} \mid T(\mathfrak{a})=\lambda(\mathfrak{a}) \mathbf{f}$ with $\lambda(\mathfrak{a}) \in \mathbb{C}$ for all integral ideals $\mathfrak{a}$. For a Hecke character $\chi$ of $F$ of conductor $\mathfrak{f}_{\chi}$ Shimura shows (see for example [10, Chapter V]) that the function (defined for $\operatorname{Re}(s)>>0$ ),

$$
\left(\prod_{i=1}^{n} L_{\mathfrak{c}}(2 s+1-2 i, \chi)\right) \sum_{\mathfrak{a}} \lambda(\mathfrak{a}) \chi^{*}(\mathfrak{a}) N(\mathfrak{a})^{-s},
$$

has an Euler product, which we denote by $Z(s, \mathbf{f}, \chi)=\prod_{\mathfrak{p}} Z_{\mathfrak{p}}\left(\chi^{*}(\mathfrak{p}) N(\mathfrak{p})^{-s}\right)$, where $\chi *$ denotes the corresponding to $\chi$ ideal class character. We will consider another $L$-function denoted by $L(s, \mathbf{f}, \chi)$ and defined as

$$
L(s, \mathbf{f}, \chi):=\prod_{\mathfrak{p}} Z_{\mathfrak{p}}\left(\chi^{*}(\mathfrak{p})\left(\psi / \psi_{\mathfrak{c}}\right)\left(\pi_{\mathfrak{p}}\right) N(\mathfrak{p})^{-s}\right),
$$

where $\pi_{\mathfrak{p}}$ is a uniformizer of the prime ideal $\mathfrak{p}$.

For $\tau \in S_{+} \cap G L_{n}(F)$ and $r \in G L_{n}(F)_{\mathbf{f}}$ we define the Dirichlet series,

$$
D_{r, \tau}(s, \mathbf{f}, \chi):=\sum_{x \in B / E} \psi(\operatorname{det}(r x)) \chi(\operatorname{det}(x)) c(\tau, r x ; \mathbf{f})|\operatorname{det}(x)|_{F}^{s-n-1},
$$

where $B=G L_{n}(F)_{\mathbf{f}} \cap \prod_{v \in \mathbf{f}} M_{n}\left(\mathcal{O}_{v}\right)$ and $E=\prod_{v \in \mathbf{f}} G L_{n}\left(\mathcal{O}_{v}\right)$. By Theorem [10, Theorem 21.4] (and the notation there for $\mathbf{b}, \mu(\cdot), \mathcal{L}_{\tau}, g_{v}$ ), we have the Andrianov type identity,

$$
\begin{aligned}
& \left(\psi / \psi_{\mathfrak{c}}\right)(\operatorname{det}(r))^{-2} D_{r, \tau}(s, \mathbf{f}, \chi) \Lambda_{\mathfrak{c}}\left(\frac{2 s-n}{4}\right) \prod_{v \in \mathbf{b}} g_{v}\left(\chi\left(\psi / \psi_{\mathfrak{c}}\right)\left(\pi_{v}\right)\left|\pi_{v}\right|^{s}\right) \\
& \quad=L(s, \mathbf{f}, \chi) \sum_{L<M \in \mathcal{L}_{\tau}} \mu(M / L)\left(\psi_{\mathfrak{c}}^{2} / \psi\right)(\operatorname{det}(y)) \chi\left(\operatorname{det}\left({ }^{t} r^{t} y^{-1}\right) \mathcal{O}\right) \mid \operatorname{det}\left(\left.{ }^{t} r^{t} y^{-1}\right|_{F} ^{s} c(\tau, y ; \mathbf{f}),\right.
\end{aligned}
$$

where for an integral ideal $\mathfrak{a}$ we write

$$
\Lambda_{\mathfrak{a}}(s)= \begin{cases}L_{\mathfrak{a}}\left(2 s, \rho_{\tau} \psi \chi\right) \prod_{i=1}^{n / 2} L_{\mathfrak{a}}\left(4 s-2 i, \psi^{2} \chi^{2}\right), & \text { if } n \text { is odd } \\ \prod_{i=1}^{(n+1) / 2} L_{\mathfrak{a}}\left(4 s-2 i+1, \psi^{2} \chi^{2}\right), & \text { if } n \text { is even. }\end{cases}
$$

We now write $t^{\prime} \in \mathbb{Z}^{d}$ for the sign

$$
(\psi \chi)_{\infty}(x)=x_{\infty}^{-t^{\prime}}\left|x_{\infty}\right|^{t^{\prime}}
$$

and define $\mu \in \mathbb{Z}^{d}$ by the conditions $0 \leq \mu_{v} \leq 1$ for all $v \in \infty$ and $\mu-[k]-t^{\prime} \in 2 \mathbb{Z}^{d}$. We moreover define the weight $l:=\mu+(n / 2) u$, and write $\theta_{\chi} \in \mathcal{M}_{l}\left(C^{\prime}, \chi^{-1} \rho_{\tau}\right)$ for the theta series associated to the data $\left(\chi^{-1}, \mu, \tau, r\right)$ in [10, Proposition A3.19], where $\rho_{\tau}$ is the Hecke character of $F$ corresponding to the extension $F\left(c^{\frac{1}{2}}\right) / F$ with $c:=(-1)^{[n / 2]} \operatorname{det}(2 \tau)$. After writing $\left.C^{\prime}=D\left[\mathfrak{b}^{\prime-1}, \mathfrak{b}^{\prime} \mathfrak{c}^{\prime}\right]\right\}$, we define $\mathfrak{e}:=\mathfrak{b}+\mathfrak{b}^{\prime}$. Then we have (see [8, pp. 342-343]),

Theorem 4.1 (Shimura)

$$
\begin{aligned}
(4 \pi)^{-n(s u+(k+l) / 2)}\left(\sqrt{D_{F}} N(\mathfrak{e})^{-1}\right)^{n(n+1) / 2} \prod_{v \in \infty} \Gamma_{n}\left(s+\left(k_{v}+l_{v}\right) / 2\right) \\
\\
D_{r, \tau}(2 s+3 n / 2+1 ; \mathbf{f}, \chi) \\
=\beta(s) \int_{\mathcal{F}} f(z) \overline{\theta_{\chi}(z) E\left(z, \bar{s}+(n+1) / 2, k-l, \overline{\psi \chi \rho_{\tau}}, \Gamma^{\prime}\right)} \delta(z)^{k} d z,
\end{aligned}
$$

where $\beta(s):=|\operatorname{det}(r)|_{F}^{-2 s-n / 2} \operatorname{det}(\tau)^{(k+\mu+n u / 2) / 2+s u}, \Gamma^{\prime}:=G \cap D\left[\mathfrak{e}^{-1}, \mathfrak{e h}\right]$, with $\mathfrak{h}=$ $\mathfrak{e}^{-1}\left(\mathfrak{b} \mathfrak{c} \cap \mathfrak{b}^{\prime} \mathfrak{c}^{\prime}\right)$, and $\mathcal{F}=\mathcal{H} / \Gamma^{\prime}$. 
In particular using the Eq. 4.2 we obtain

Theorem 4.2 (Shimura)

$$
\begin{aligned}
\left.\psi / \psi_{\mathfrak{c}}\right)^{2}\left((\operatorname{det}(r)) L(s, \mathbf{f}, \chi) \prod_{v \in \infty} \Gamma_{n}\left(\frac{s-n-1+k_{v}+\mu_{v}}{2}\right)\right. \\
\quad \times \sum_{L<M \in \mathcal{L}_{\tau}} \mu(M / L)\left(\psi_{\mathfrak{c}}^{2} / \psi\right)(\operatorname{det}(y)) \chi\left(\operatorname{det}\left({ }^{t} r^{t} y^{-1}\right)\right)\left|\operatorname{det}\left({ }^{t} r^{t} y^{-1}\right)\right|_{F}^{s} c(\tau, y ; \mathbf{f}) \\
=\left(D_{F}^{-1 / 2} N(\mathfrak{e})\right)^{n(n+1) / 2}(4 \pi)^{n d s^{\prime}+\frac{n}{2} \sum_{v}\left(k_{v}+\mu_{v}+n / 2\right)} \operatorname{det}(\tau)^{s^{\prime} u+\frac{k+\mu+n / 2 u}{2}}|\operatorname{det}(r)|_{F}^{n+1-s} \\
\quad \times \prod_{v \in \mathbf{b}} g_{v}\left(\left(\psi / \psi_{\mathbf{c}}\right)\left(\pi_{v}\right) \chi\left(\pi_{v}\right)\left|\pi_{v}\right|^{s}\right)\left(\Lambda_{\mathfrak{c}} / \Lambda_{\mathfrak{h}}\right)((2 s-n) / 4) \operatorname{vol}(\mathcal{F}) \\
\quad<f, \theta_{\chi} D((2 s-n) / 4)>
\end{aligned}
$$

where $s^{\prime}=(2 s-3 n-2) / 4$, and $D(s)=\overline{\Lambda_{\mathfrak{h}}(s)} E\left(z, \bar{s} ; k-l, \rho_{\tau} \overline{\psi \chi}, \Gamma\right)$, and

$$
<f, \theta_{\chi} D((2 s-n) / 4)>=\operatorname{vol}(\mathcal{F})^{-1} \int_{\mathcal{F}} f(z) \overline{\theta_{\chi}(z) D(z,(2 s-n) / 4)} \delta(z)^{k} d z .
$$

In particular there exists $(\tau, r)$ with $c(\tau, r ; \mathbf{f}) \neq 0$ such that

$$
\begin{aligned}
& L(s, \mathbf{f}, \chi) \prod_{v \in \infty} \Gamma_{n}\left(\frac{s-n-1+k_{v}+\mu_{v}}{2}\right) \psi_{\mathfrak{c}}(\operatorname{det}(r)) c(\tau, r ; \mathbf{f}) \\
& =\left(D_{F}^{-1 / 2} N(\mathfrak{e})\right)^{n(n+1) / 2}(4 \pi)^{n d s^{\prime}+\frac{n}{2} \sum_{v}\left(k_{v}+\mu_{v}+n / 2\right)} \operatorname{det}(\tau)^{s^{\prime} u+\frac{k+\mu+n / 2 u}{2}}|\operatorname{det}(r)|_{F}^{n+1-2 s} \\
& \quad \times \prod_{v \in \mathbf{b}} g_{v}\left(\left(\psi / \psi_{\mathbf{c}}\right)\left(\pi_{v}\right) \chi\left(\pi_{v}\right)\left|\pi_{v}\right|^{s}\right)\left(\Lambda_{\mathfrak{c}} / \Lambda_{\mathfrak{h}}\right)((2 s-n) / 4) \operatorname{vol}(\mathcal{F}) \\
& \quad<f, \theta_{\chi} D((2 s-n) / 4)>.
\end{aligned}
$$

\section{Petersson inner products and periods}

In this section we define some archimedean periods that we will use to normalize the special values of the function $L(s, \mathbf{f}, \psi)$. We start by proving a lemma with respect to the action of "good" Hecke operator $T(\mathfrak{a})$, relative to the group $C=D\left[\mathfrak{b}^{-1}, \mathfrak{b} c\right]$. Here "good" means that $\mathfrak{a}$ is prime to $\mathfrak{c}$.

Lemma 5.1 The Hecke operators $T(\mathfrak{a})$, preserve $\mathcal{M}_{k}(C, \psi, W)$, if $W$ contains the values of $\psi$.

Proof Following Shimura in [7, p. 44] we consider the formal Dirichlet series $\mathbf{f} \mid \mathfrak{I}:=\sum_{\mathfrak{a}}$ $[\mathfrak{a}] \mathbf{f} \mid T(\mathfrak{a})$ (Our notation here is slightly different from the one of Shimura). For a $\tau \in S_{+}$and $q \in G L_{n}(F)_{\mathbf{f}}$ Shimura shows in (p. 46, loc. cit.) that

$c(\tau, q ; \mathbf{f} \mid \Im)=\sum_{g, h} \psi_{\mathfrak{c}}\left(\operatorname{det}\left(h^{-1} g\right)\right)|\operatorname{det}(g)|_{\mathbb{A}}^{-n-1} c\left(\tau, q h^{-1} g ; \mathbf{f}\right) \alpha^{\prime}\left({ }^{t} h^{-1} t q \tau q h^{-1}\right)[\operatorname{det}(g h) \mathcal{O}]$.

For the notation we refer to Shimura's paper. The point which is important here is that by Proposition 4.1 in (loc. cit.) we have that $\alpha^{\prime}(\cdot)$ is a rational formal Dirichlet series (i.e. has coefficients in $\mathbb{Q})$. In particular by the equation above we conclude that the $c(\tau, q ; \mathbf{f} \mid T(\mathfrak{a}))$, which is obtained by equating the $[\mathfrak{a}]$ coefficient in the formal Dirichlet series, is a $\mathbb{Q}(\psi)$ linear combination of the Fourier coefficients of $\mathbf{f}$. Hence we conclude the lemma. 
In the following we write $<\cdot, \cdot>$ for the adelic Petersson inner product (see for example [9] for the definition) and we denote by $\mathcal{S}_{k}(\overline{\mathbb{Q}})$ the space of weight $k$ cusp forms with algebraic Fourier coefficients and of any congruence subgroup.

Theorem 5.2 Let $\mathbf{f} \in \mathcal{S}_{k}(C, \psi)$ be an eigenform for all the good Hecke operators of $C$. Let $\Phi$ be the Galois closure of $F$ over $\mathbb{Q}$ and write $\Psi$ for extension of $\Phi$ generated by the Fourier coefficients of $\mathbf{f}$ and their complex conjugation. Assume $m_{0}:=\min _{v}\left(k_{v}\right)>[3 n / 2+1]+2$. Then there exists a period $\Omega_{\mathbf{f}} \in \mathbb{C}^{\times}$and a finite extension $L$ of $\Phi$ such that for any $\mathbf{g} \in \mathcal{S}_{k}(\overline{\mathbb{Q}})$ we have

$$
\left(\frac{<\mathbf{f}, \mathbf{g}>}{\Omega_{\mathbf{f}}}\right)^{\sigma}=\frac{<\mathbf{f}^{\sigma}, \mathbf{g}^{\sigma^{\prime}}>}{\Omega_{\mathbf{f}^{\sigma}}},
$$

for all $\sigma \in \operatorname{Gal}(\overline{\mathbb{Q}} / L)$, where $\sigma^{\prime}=\rho \sigma \rho$. Moreover $\Omega_{\mathbf{f}}$ depends only on the eigenvalues of f and we have $\frac{<\mathbf{f}, \mathbf{f}>}{\Omega_{\mathbf{f}}} \in(L \Psi)^{\times}$.

Remark 5.3 We make the following two remarks,

(1) Our proof is using ideas from a result of Sturm [11, Theorem 3], and of Shimura [10, Theorem 28.5]. We have similar theorems in the integral weight situation [1]. The proof is very similar to integral weight situation considered in [1], and hence we will not reproduce all the long calculations again, but only what is needed to have a logical flow and we simply refer to our previous work for the details. Perhaps the differences that deserve special mention are (a) the fact that we need to establish the Galois invariance of the trace map for half-integral Siegel modular forms (see Lemma 5.4 below), and (b) the slightly larger extension $L$.

(2) Indeed the number field $L$ in the statement of the theorem, depends only the eigenspace

$$
\mathcal{V}:=\left\{\mathbf{g} \in \mathcal{S}_{k}(C, \psi)|\mathbf{g}| T(\mathfrak{a})=\lambda_{\mathbf{f}}(\mathfrak{a}) \mathbf{g}(\mathfrak{a}, \mathfrak{c})=1\right\},
$$

where $\left\{\lambda_{\mathbf{f}}(\mathfrak{a})\right\}$ are the eigenvalues of $\mathbf{f}$, with respect to the good Hecke operators. $L$ is the composition of $\Phi$ with quadratic extensions of $\mathbb{Q}$ and a rough estimation shows that $[L ; \Phi] \leq 2^{\ell-1}$ where $\ell$ is the dimension of $\mathcal{V}$.

Proof As in [1] we pick a half integer $\sigma_{0}$ so that $3 n / 2+1<\sigma_{0}<m_{0}$ and $m_{0}-\sigma_{0} \notin 2 \mathbb{Z}$ and define $\mu \in \mathbb{Z}^{d}$ by the conditions $0 \leq \mu_{v} \leq 1$ and $\sigma_{0}-k_{v}+\mu_{v} \in 2 \mathbb{Z}$ for all $v \in \infty$. Our choice of $\sigma_{0}$ implies in particular that there exists an $v \in \infty$ so that $\mu_{v} \neq 0$. We put $t^{\prime}:=\mu-k$. We now pick a quadratic character $\chi$ of $F$ so that $(\psi \chi)_{\infty}(x)=x_{\infty}^{t^{\prime}}\left|x_{\infty}\right|^{-t^{\prime}}$ and of conductor $\mathfrak{f}$ such that $\mathfrak{c} \mid \mathfrak{f}$ (see [1] for the existence of such an $\chi$ ) and we define $l:=\mu+(n / 2) u$ and $v=\sigma_{0}-(n / 2)$. Then $v \geq(n+1) / 2$ and $0 \leq k-l-v u \in 2 \mathbb{Z}^{d}$. We consider the theta series $\theta_{\chi}$ with respect to our choices of $\chi$ and $\mu$, and by our choices we note that $\theta_{\chi}$ is a cusp form, since $\mu \neq 0$ (see [10]). By Theorem 4.2 we have

$$
\begin{aligned}
& L\left(\sigma_{0}, \mathbf{f}, \chi\right) \prod_{v \in \infty} \Gamma_{n}\left(\frac{\sigma_{0}-n-1+k_{v}+\mu_{v}}{2}\right)\left(\psi / \psi_{\mathfrak{c}}\right)^{2}(\operatorname{det}(r)) \\
& \quad \times \sum_{L<M \in \mathcal{L}_{\tau}} \mu(M / L)\left(\psi_{\mathfrak{c}}^{2} / \psi\right)(\operatorname{det}(y)) \chi\left(\operatorname{det}\left({ }^{t} r^{t} y^{-1}\right)\right)\left|\operatorname{det}\left({ }^{t} r^{t} y^{-1}\right)\right|_{F}^{\sigma_{0}} c(\tau, y ; \mathbf{f}) \\
& =\left(D_{F}^{-1 / 2} N(\mathfrak{e})\right)^{n(n+1) / 2}(4 \pi)^{n d s_{0}^{\prime}+\frac{n}{2} \sum_{v}\left(k_{v}+\mu_{v}+n / 2\right)} \operatorname{det}(\tau)^{s_{0}^{\prime} u+\frac{k+\mu+n / 2 u}{2}}|\operatorname{det}(r)|_{F}^{n+1-\sigma_{0}} \\
& \quad \times \prod_{v \in \mathbf{b}} g_{v}\left(\left(\psi / \psi_{\mathbf{c}}\right)\left(\pi_{v}\right) \chi\left(\pi_{v}\right)\left|\pi_{v}\right|^{\sigma_{0}}\right)\left(\Lambda_{\mathfrak{c}} / \Lambda_{\mathfrak{h}}\right)(v / 2) \operatorname{vol}(\mathcal{F})<f, \theta_{\chi} D\left(v / 2, \rho_{\tau} \overline{\psi \chi}\right)>,
\end{aligned}
$$

where $s_{0}^{\prime}=\left(2 \sigma_{0}-3 n-2\right) / 4$. 
We write $\delta$ for the rational part of $\frac{\prod_{v \in \infty} \Gamma_{n}\left(\frac{\sigma_{0}-n-1+k_{v}+\mu_{v}}{2}\right)}{\operatorname{vol}(\mathcal{F})}$ and define $\beta \in \mathbb{N}$ so that $\pi^{-\beta} D(v / 2) \in \mathcal{N}_{k-l}^{p}\left(\Phi \mathbb{Q}_{a b}\right)$ with $p=\frac{k-l-v u}{2}$. We set $\gamma:=n\left(\sum_{v} \frac{k_{v}-l_{v}-v}{2}-k_{v}\right)+d \epsilon-$ $n \sum_{v}\left(s_{0}^{\prime}+k_{v}+\mu_{v}+n / 2\right)-\beta$, where $\epsilon=n^{2} / 4$ if $n$ even and $\left(n^{2}-1\right) / 4$ otherwise. Similar to [1] we define,

$$
\begin{aligned}
& B(\chi, \psi, \tau, r, \mathbf{f}):=\delta|\operatorname{det}(r)|_{\infty}^{n+1-\sigma_{0}}\left(\psi / \psi_{\mathfrak{c}}\right)^{2}(\operatorname{det}(r)) \\
& \quad \times \sum_{L<M \in \mathcal{L}_{\tau}} \mu(M / L)\left(\psi_{\mathfrak{c}}^{2} / \psi\right)(\operatorname{det}(y)) \chi\left(\operatorname{det}\left({ }^{t} r^{t} y^{-1}\right)\right)\left|\operatorname{det}\left({ }^{t} r^{t} y^{-1}\right)\right|_{F}^{\sigma_{0}} c(\tau, y ; \mathbf{f}),
\end{aligned}
$$

and

$$
C(\chi, \psi, \tau, r):=(N(\mathfrak{e}))^{n(n+1) / 2} \prod_{v \in \mathbf{b}} g_{v}\left(\left(\psi / \psi_{\mathbf{c}}\right)\left(\pi_{v}\right) \chi\left(\pi_{v}\right)\left|\pi_{v}\right|^{\sigma_{0}}\right)\left(\Lambda_{\mathfrak{c}} / \Lambda_{\mathfrak{h}}\right)(v / 2) .
$$

We now claim that for every $\sigma \in \operatorname{Gal}(\overline{\mathbb{Q}} / \Phi)$ we have that

$$
B(\chi, \psi, \tau, r, \mathbf{f})^{\sigma}=B\left(\chi^{\sigma}, \psi^{\sigma}, \tau, r, \mathbf{f}^{\sigma}\right) \text { and } C(\chi, \psi, \tau, r)^{\sigma}=C\left(\chi^{\sigma}, \psi^{\sigma}, \tau, r\right) .
$$

For $C(\chi, \psi, \tau, r)$ this is easy to see, since $\sigma_{0}+\frac{1}{2}$ is an integer, and hence our claim follows by looking at the definition of $g_{v}$ in [10, p. 128]. However for $B(\chi, \psi, \tau, r, \mathbf{f})$ we need to justify it since $\sigma_{0} \in \mathbb{Z}+\frac{1}{2}$. For this we rewrite

$$
\begin{aligned}
& B(\chi, \psi, \tau, r, \mathbf{f})=\delta|\operatorname{det}(r)|_{F}^{n+1}\left(\psi / \psi_{\mathfrak{c}}\right)^{2}(\operatorname{det}(r)) \\
& \quad \times \sum_{L<M \in \mathcal{L}_{\tau}} \mu(M / L)\left(\psi_{\mathfrak{c}}^{2} / \psi\right)(\operatorname{det}(y)) \\
& \quad \chi\left(\operatorname{det}\left({ }^{t} r^{t} y^{-1}\right)\right)\left|\operatorname{det}\left({ }^{t} y^{-1}\right)\right|_{F}^{\sigma_{0}+\frac{1}{2}}\left|\operatorname{det}\left({ }^{t} y^{-1}\right)\right|_{F}^{-\frac{1}{2}} c(\tau, y ; \mathbf{f}) .
\end{aligned}
$$

We recall that $\mu(\tau, y ; \mathbf{f})=\left|\operatorname{det}\left({ }^{t} y^{-1}\right)\right|_{F}^{-\frac{1}{2}} c(\tau, y ; \mathbf{f})$, and by [10, Lemma 23.14] we have that $\mu(\tau, y ; \mathbf{f})^{\sigma}=\mu\left(\tau, y ; \mathbf{f}^{\sigma}\right)$, from which the claim follows.

Writing $\operatorname{Tr}_{\Gamma^{\prime}}^{\Gamma}: \mathcal{S}_{k}\left(\Gamma^{\prime}, \psi\right) \rightarrow S_{k}(\Gamma, \psi)$ for the usual trace operator attached to the groups $\Gamma^{\prime} \leq \Gamma$, and using the properties of the inner product we obatin

$$
<f, \theta_{\chi} D\left(v / 2, \rho_{\tau} \overline{\psi \chi}\right)>=<f, \mathfrak{p}\left(\theta_{\chi} D\left(v / 2, \rho_{\tau} \overline{\psi \chi}\right)\right)>=<f, \operatorname{Tr}_{\Gamma^{\prime}}^{\Gamma}\left(\mathfrak{p}\left(\theta_{\chi} D\left(v / 2, \rho_{\tau} \overline{\psi \chi}\right)\right)>,\right.
$$

where $\mathfrak{p}: \mathcal{R}_{k}^{p} \rightarrow \mathcal{S}_{k}$ is Shimura's holomorphic projection operators [10, Proposition 15.6]. Moreover, since $\theta_{\chi} \pi^{-\beta} D(v / 2) \in \mathcal{N}_{k}^{p}\left(\Phi \mathbb{Q}_{a b}\right)$, we may consider the action of $\sigma \in \operatorname{Gal}\left(\Phi \mathbb{Q}_{a b} / \Phi\right)$. Then

$$
\mathfrak{p}\left(\theta_{\chi} \pi^{-\beta} D\left(v / 2, \rho_{\tau} \overline{\psi \chi}\right)\right)^{\sigma}=\mathfrak{p}\left(\theta_{\chi}^{\sigma}\left(\pi^{-\beta} D\left(\nu / 2, \rho_{\tau} \overline{\psi \chi}\right)\right)^{\sigma}\right),
$$

and

$$
\operatorname{Tr}_{\Gamma^{\prime}}^{\Gamma}\left(\theta_{\chi} \pi^{-\beta} D\left(v / 2, \rho_{\tau} \overline{\psi \chi}\right)\right)^{\sigma}=\operatorname{Tr}_{\Gamma^{\prime}}^{\Gamma}\left(\theta_{\chi}^{\sigma} D\left(v / 2, \rho_{\tau} \overline{\psi \chi}\right)^{\sigma}\right),
$$

using an equivariant property of the trace, which will be shown below in Lemma 5.4. Now, for any given $\mathbf{f} \in \mathcal{V}$, there exists $(\tau, r)$ such that

$$
B(\chi, \psi, \tau, r, \mathbf{f})=\delta \psi(\operatorname{det}(r)) c(\tau, r ; \mathbf{f}) \neq 0,
$$

and we write $\mathfrak{G}$ for the set of pairs $(\tau, r)$ for which such an $\mathbf{f}$ exists. In particular for such an $(\tau, r)$, we have (see [1] for details) that

$$
<f, \theta_{\chi} \pi^{-\beta} D\left(v / 2, \rho_{\tau} \overline{\psi \chi}\right)>\neq 0 \text {, and } C(\chi, \psi, \tau, r) \neq 0 .
$$


We now define $\mathbf{g}_{\tau, r} \in \mathcal{S}_{k}\left(\Gamma^{\prime}, \bar{\psi} ; \Phi \mathbb{Q}_{a b}\right)$ by

$$
\mathbf{g}_{\tau, r}=\operatorname{Tr}_{\Gamma^{\prime}}^{\Gamma}\left(\mathfrak{p}\left(\theta_{\chi} \pi^{-\beta} D\left(v / 2, \rho_{\tau} \overline{\psi \chi}\right)\right)\right)
$$

and define the space $\mathcal{W}$ to be the space generated by $\mathbf{g}_{\tau, r}$ for $(\tau, r) \in \mathfrak{G}$. We now claim that there exists an $\Omega_{\mathbf{f}} \in \mathbb{C}^{\times}$such that any $\mathbf{f} \in \mathcal{V}$ and any $\mathbf{g}_{\tau, r}$

$$
\left(\frac{<\mathbf{f}, \mathbf{g}_{\tau, r}>}{\Omega_{\mathbf{f}}}\right)^{\sigma}=\frac{<\mathbf{f}^{\sigma}, \mathbf{g}_{\tau, r}^{\sigma^{\prime}}>}{\Omega_{\mathbf{f}^{\sigma}}},
$$

where $\sigma^{\prime}=\rho \sigma \rho$, and $\sigma \in \operatorname{Gal}(\overline{\mathbb{Q}} / \Phi(\sqrt{\operatorname{det}(\tau)}))$.

The case of $n$ even This case is similar to the case of $n$ odd in the integral weight situation, which we considered in our previous work [1]. We do not reproduce the whole calculations but we refer the reader to that article. The only difference is that now we have to take the element $\sigma$ to fix $\Phi(\sqrt{\operatorname{det}(\tau)})$. The same calculations (there we take $\epsilon=1$ ) show that we can define:

$$
\Omega_{\mathbf{f}}:=\left(\overline{\mathbf{g}(\bar{\psi})}^{n} \overline{\mathbf{g}(\bar{\phi})}^{n} \bar{R}\right)^{-1} D_{F}^{n(n+1) / 4} \pi^{\gamma} L\left(\sigma_{0}, \mathbf{f}, \chi\right)
$$

where

$$
R:=i^{n|p|} i^{-d n v} C D_{F}^{\frac{n}{2}\left(\sum_{v} k_{v}-\mu_{v}-\frac{n}{2}\right)+3 n(n+1) / 4}
$$

The case of $n$ odd This case is the same with the case of $n$ even in the integral weight situation. We set:



where $B(n)=i^{d}$ if $[n / 2]$ is odd and 1 otherwise. Moreover

$$
R(\bar{\psi}):=\frac{i^{n|p|}(i)^{\nu d}}{D_{F}^{1 / 2}} \frac{\left(\prod_{i=1}^{[n / 2]}(i)^{(2 v-2 i) d}\right) \mathbf{g}\left(\bar{\psi}^{2[n / 2]}\right)}{D_{F}^{b(n)}},
$$

where $b(n)$ is defined as in Theorem 3.2.

As in [1] it can be shown that the $\mathbb{C}$ span (of the projections) of $\mathbf{g}_{\tau, r}$ with $(\tau, r) \in \mathfrak{G}$ is equal to $\mathcal{V}$. Since $\mathcal{V}$ is a finite dimensional space, we need only finitely many $\mathbf{g}_{\tau, r}$. We define $L$ to be a minimal extension of $\Phi$ such that $L$ contains $\sqrt{\operatorname{det}(\tau)}$ for a set of $\mathbf{g}_{\tau, r}$ spanning $\mathcal{V}$. Since $\mathbf{g}_{\tau, r} \in S_{k}(\overline{\mathbb{Q}})$ we have that the $\overline{\mathbb{Q}}$-span is equal to $\mathcal{V}(\overline{\mathbb{Q}})$. We can now establish the theorem for any $\mathbf{g} \in \mathcal{V}(\overline{\mathbb{Q}})$ since after writing $\mathbf{g}=\sum_{j} c_{j} \mathbf{g}_{\tau_{j}, r_{j}, \mathcal{V}} \in \mathcal{V}(\overline{\mathbb{Q}})$, where $\mathbf{g}_{\tau_{j}, r_{j}, \mathcal{V}}$ is the projection of $\mathbf{g}_{\tau_{j}, r_{j}}$ to $\mathcal{V}$, we have for all $\sigma \in \operatorname{Gal}(\overline{\mathbb{Q}} / L)$ that,

$$
\left(\frac{<\mathbf{f}, \mathbf{g}>}{\Omega_{\mathbf{f}}}\right)^{\sigma}=\sum_{j}{\overline{c_{j}}}^{\sigma}\left(\frac{<\mathbf{f}^{\sigma}, \mathbf{g}_{\tau_{j}, r_{j}, \mathcal{V}}^{\sigma^{\prime}}}{\Omega_{\mathbf{f}^{\sigma}}}\right)=\frac{<\mathbf{f}^{\sigma}, \mathbf{g}^{\sigma^{\prime}}>}{\Omega_{\mathbf{f}}{ }^{\sigma}} .
$$

We now take any $\mathbf{g} \in \mathcal{S}_{k}(\Gamma, \psi ; \overline{\mathbb{Q}})$. Since the good Hecke operators act semisimply, we have $\mathcal{S}_{k}(C, \psi)=\mathcal{V} \oplus U$, for some vector space $U$ preserved by the action of the Hecke operators. We write $\mathbf{g}=\mathbf{g}_{1}+\mathbf{g}_{2}$ with $\mathbf{g}_{1} \in \mathcal{V}$ and $\mathbf{g}_{2} \in U$. Then we have that

$$
\left(\frac{<\mathbf{f}, \mathbf{g}>}{\Omega_{\mathbf{f}}}\right)^{\sigma}=\left(\frac{<\mathbf{f}, \mathbf{g}_{1}>}{\Omega_{\mathbf{f}}}\right)^{\sigma}=\frac{<\mathbf{f}^{\sigma}, \mathbf{g}_{1}^{\sigma^{\prime}}>}{\Omega_{\mathbf{f}}}=\frac{<\mathbf{f}^{\sigma}, \mathbf{g}^{\sigma^{\prime}}>}{\Omega_{\mathbf{f}^{\sigma}}},
$$


where the first and the last equality follows from the fact that $<\mathbf{f}, \mathbf{g}>=0$ and $<\mathbf{f}^{\sigma}, \mathbf{g}^{\sigma^{\prime}}>=0$ for $\mathbf{g} \in U$. By Lemma 5.1 it is enough to prove this for $\mathbf{g}$ an eigenform for all the good Hecke operators with a system of eigenvalues different from that of $\mathbf{f}$ 's. That is, there exists an ideal $\mathfrak{a}$ with $(\mathfrak{a}, \mathfrak{c})=1$ so that $T(\mathfrak{a}) \mathbf{f}=\lambda_{\mathbf{f}}(\mathfrak{a}) \mathbf{f}$ and $T(\mathfrak{a}) \mathbf{g}=\lambda_{\mathbf{g}}(\mathfrak{a}) \mathbf{g}$ such that $\lambda_{\mathbf{f}}(\mathfrak{a}) \neq \lambda_{\mathbf{g}}(\mathfrak{a})$. By [10, Lemma 23.14] we have that $T(\mathfrak{a}) \mathbf{f}^{\sigma}=\lambda_{\mathbf{f}}(\mathfrak{a})^{\sigma} \frac{\left(N(\mathfrak{a})^{\frac{1}{2}}\right)^{\sigma}}{N(\mathfrak{a})^{\frac{1}{2}}} \mathbf{f}^{\sigma}$, and similarly for $\mathbf{g}$. But then we have

$$
\begin{aligned}
& \lambda_{\mathbf{f}}(\mathfrak{a})^{\sigma} \frac{N\left(\mathfrak{a}^{\frac{1}{2}}\right)^{\sigma}}{N(\mathfrak{a})^{\frac{1}{2}}}<\mathbf{f}^{\sigma}, \mathbf{g}^{\sigma^{\prime}}>=<T(\mathfrak{a}) \mathbf{f}^{\sigma}, \mathbf{g}^{\sigma^{\prime}}>= \\
& \quad<\mathbf{f}^{\sigma}, T(\mathfrak{a}) \mathbf{g}^{\sigma^{\prime}}>=<\mathbf{f}^{\sigma}, \lambda_{\mathbf{g}}(\mathfrak{a})^{\sigma^{\prime}} \frac{\left.N(\mathfrak{a})^{\frac{1}{2}}\right)^{\sigma^{\prime}}}{N(\mathfrak{a})^{\frac{1}{2}}} \mathbf{g}^{\sigma^{\prime}}>=<\mathbf{f}^{\sigma}, \mathbf{g}^{\sigma^{\prime}}>\lambda_{\mathbf{g}}(\mathfrak{a})^{\sigma} \frac{\left.N(\mathfrak{a})^{\frac{1}{2}}\right)^{\sigma}}{N(\mathfrak{a})^{\frac{1}{2}}}
\end{aligned}
$$

and hence we conclude that $<\mathbf{f}^{\sigma}, \mathbf{g}^{\sigma^{\prime}}>=0$. Here we have used the facts that the good Hecke operators are self adjoint with respect to the Petersson inner product, and that their Hecke eigenvalues are totally real (for both facts see [10, Lemma 23.15]).

Finally taking $\mathbf{g}$ equal to $\mathbf{f}$ we obtain that $\Omega_{\mathbf{f}}$ is equal to $<\mathbf{f}, \mathbf{f}>$ up to a non-zero element $\Psi L$.

In order to complete the proof we need to establish the result on the Galois equivariance of the trace operator. We will need the theta series $\theta(z):=\sum_{a \in \mathcal{O}^{n}} \mathbf{e}_{\infty}\left({ }^{t} a z a / 2\right) \in \mathcal{M}_{\frac{1}{2} u}(\mathbb{Q})$. Note that this is the series $\theta_{F}$ defined in [10, p. 39, equation 6.16] by taking in the equation there, using Shimura's notation, $u=0$ and $\lambda$ the characteristic function of $\mathcal{O}^{n} \subset F^{n}$. Note in particular that since we are taking $u=0$ we have that $\phi_{F}=\theta_{F}$. In particular Theorem 6.8 in (loc. cit) gives the properties of the series $\theta$.

The following lemma is an extension of a result of Sturm in [11, Lemma 11] to the half-integral weight situation. The following proof is an extension of our previous proof [1, Lemma 8]. The key difference is the use of the theta series $\theta$ to go from the half-integral weight situation to the integral weight situation.

Lemma 5.4 For any $f \in \mathcal{S}_{k}\left(\Gamma^{\prime}, \psi ; \mathbb{Q}_{a b}\right)$

$$
\operatorname{Tr}_{\Gamma^{\prime}, \psi}^{\Gamma}(f)^{\sigma}=\operatorname{Tr}_{\Gamma^{\prime}, \psi^{\sigma}}^{\Gamma}\left(f^{\sigma}\right), \quad \sigma \in \operatorname{Gal}\left(\Phi \mathbb{Q}_{a b} / \Phi\right)
$$

Proof In the proof of this lemma we will make use of Shimura's reciprocity law of the action of $G_{\mathbb{A}}$ to the space of integral weight Siegel modular forms. We will use the notation as in [10, Theorem 10.2].

Since $k \notin \mathbb{Z}^{d}$, we have that $\theta f \in \mathcal{M}_{k^{\prime}}$, with $k^{\prime}=k+\frac{1}{2} \in \mathbb{Z}^{d}$ and of Nebentypus $\psi \phi$, where $\phi$ is as in Theorem 3.2. Moreover for any $\sigma \in \operatorname{Gal}\left(\frac{\mathbb{Q}}{\mathbb{Q}}\right)$ we have that $(\theta f)^{\sigma}=\theta f^{\sigma}$ since $\theta$ has Fourier expansion with coefficients in $\mathbb{Q}$. We moreover observe that $\theta \operatorname{Tr}_{\Gamma^{\prime}, \psi}^{\Gamma}(f)=$ $\operatorname{Tr}_{\Gamma^{\prime}, \psi \phi}^{\Gamma}(\theta f)$ sicne we have $\left.\theta\right|_{\frac{1}{2} u} \gamma=\theta$ for all $\gamma \in \Gamma \subseteq \Gamma^{\theta}$.

Thanks to the strong approximation for $S p_{n}(F)$ we may work adelically. We write $D$ and $D^{\prime}$ for the corresponding to $\Gamma$ and $\Gamma^{\prime}$ adelic groups (i.e. $\Gamma=G \cap D$ ). We fix elements $\left\{g_{i}\right\} \subset D_{\mathbf{f}}$ so that $D=\bigcup D^{\prime} g_{i}$. For $t \in \mathbb{Z}_{\mathbf{f}}^{\times}$corresponding to $\left.\sigma\right|_{\mathbb{Q}_{a b}}$ we note that

$$
\left(\begin{array}{cc}
I_{n} & 0 \\
0 & t^{-1} I_{n}
\end{array}\right) g_{i}\left(\begin{array}{cc}
I_{n} & 0 \\
0 & t I_{n}
\end{array}\right) \in S p_{n}(\mathbb{A})_{\mathbf{f}}
$$


and hence by strong approximation we can find elements $u_{i} \in D^{\prime}$ with $f \mid u_{i}=f$ (i.e. $\left.\psi\left(u_{i}\right)=1\right), \phi\left(u_{i}\right)=1$, and $w_{i} \in S p_{n}(F)$ so that

$$
\left(\begin{array}{cc}
I_{n} & 0 \\
0 & t^{-1} I_{n}
\end{array}\right) g_{i}\left(\begin{array}{cc}
I_{n} & 0 \\
0 & t I_{n}
\end{array}\right)=u_{i} w_{i}
$$

As in [1] we can show that since the $g_{i}$ 's form a set of representatives of the classes of $D^{\prime}$ in $D$, the same holds for $\left(\begin{array}{cc}I_{n} & 0 \\ 0 & t^{-1} I_{n}\end{array}\right) g_{i}\left(\begin{array}{cc}I_{n} & 0 \\ 0 & t I_{n}\end{array}\right)$, and hence also for $w_{i}$ since $u_{i} \in D^{\prime}$. We now consider the elements $(l(t), \sigma),\left(w_{i}, i d\right),\left(g_{i}, i d\right) \in \mathcal{G}_{+} \times \operatorname{Gal}(\overline{\mathbb{Q}} / \mathbb{Q})$. Then we have

$$
\begin{aligned}
& \left(\theta \operatorname{Tr}_{\Gamma^{\prime}, \psi^{\sigma}}^{\Gamma}\left(f^{\sigma}\right)\right)^{\sigma^{-1}}=\left(\sum_{i} \psi \phi\left(g_{i}\right)^{\sigma} \theta f^{\sigma} \mid g_{i}\right)^{\sigma^{-1}} \\
& =\sum_{i} \psi \phi\left(g_{i}\right)(\theta f)\left|\left((l(t), \sigma)\left(g_{i}, 1\right)\left(\imath\left(t^{-1}\right), \sigma^{-1}\right)\right)=\sum_{i} \phi \psi\left(g_{i}\right)(\theta f)\right|\left(u_{i} w_{i}, 1\right) \\
& =\sum_{i} \psi \phi\left(g_{i}\right)(\theta f)\left|w_{i}=\sum_{i}(\psi \phi)\left(w_{i}\right)(\theta f)\right| w_{i}=\theta \sum_{i} \psi\left(w_{i}\right) f \mid w_{i}=\theta \operatorname{Tr}_{\Gamma^{\prime}, \psi}^{\Gamma} f .
\end{aligned}
$$

So we conclude that $\left.\theta \operatorname{Tr}_{\Gamma^{\prime}, \psi^{\sigma}}^{\Gamma}\left(f^{\sigma}\right)\right)^{\sigma^{-1}}=\theta \operatorname{Tr}_{\Gamma^{\prime}, \psi}^{\Gamma} f$. But $\theta$ is a non-zero divisor (see [10, p. 74]. Hence we conclude the lemma.

\section{Algebraicity results}

In this section we present various results regarding special values of the function $L(s, \mathbf{f}, \chi)$, with $\mathbf{f} \in \mathcal{S}_{k}(C, \psi), C=D\left[\mathfrak{b}^{-1}, \mathfrak{b} \mathfrak{c}\right]$, and an eigenform for all Hecke operators. We recall that we have also considered the function $Z(s, \mathbf{f}, \chi)$. For a comparison between the two we refer to [1] where the similar situation of the integral weight case is discussed.

For completeness we start by recalling the result of Shimura [10, Theorem 28.8] regarding algebraicity of special values. We take an $0 \neq \mathbf{f} \in \mathcal{S}_{k}(C ; \overline{\mathbb{Q}})$, where

$$
C=\left\{x \in D\left[\mathfrak{b}^{-1}, \mathfrak{b} \mathfrak{c}\right] \mid a_{x}-1 \prec \mathfrak{c}\right\} .
$$

We assume that $\mathbf{f}$ is an eigenform for all Hecke operators $T(\mathfrak{a})$ and we note that the $T\left(\mathfrak{q}^{m}\right)$ 's are trivial for primes $\mathfrak{q}$ and $m \in \mathbb{N}$ such that $\mathfrak{q} \mid \mathfrak{c}$.

Theorem 6.1 [10, Theorem 28.8] With notation as above define $m_{0}:=\min \left\{k_{v} \mid v \in \infty\right\}$ and assume $m_{0}>(3 n / 2)+1$. Let $\chi$ be a character of $F$ such that $\chi_{\infty}(x)=x_{\infty}^{t}\left|x_{\infty}\right|^{-t}$ with $t \in \mathbb{Z}^{d}$. Set $\mu_{v}:=0$ if $\left[k_{v}\right]-t_{v} \in 2 \mathbb{Z}$ and $\mu_{v}=1$ if $\left[k_{v}\right]-t_{v} \notin 2 \mathbb{Z}$. Let $\sigma_{0} \in \frac{1}{2} \mathbb{Z}$ such that

(1) $2 n+1-k_{v}+\mu_{v} \leq \sigma_{0} \leq k_{v}-\mu_{v}$,

(2) $\sigma_{0}-k_{v}+\mu_{v} \in 2 \mathbb{Z}$ for every $v \in \infty$ if $\sigma_{0}>n$,

(3) $\sigma_{0}-1+k_{v}-\mu_{v} \in 2 \mathbb{Z}$ for every $v \in \infty$ if $\sigma_{0} \leq n$.

We exclude the cases

(1) $\sigma_{0}=n+3 / 2, F=\mathbb{Q}, \chi^{2}=1$ and $[k]-t \in 2 \mathbb{Z}$,

(2) $0<\sigma_{0} \leq n, \mathfrak{c}=\mathcal{O}, \chi^{2}=1$ and the conductor of $\chi$ is $\mathcal{O}$.

Then,

$$
\frac{Z\left(\sigma_{0}, \mathbf{f}, \chi\right)}{<\mathbf{f}, \mathbf{f}>} \in \pi^{n\left(\sum_{v} k_{v}\right)+d\left(n \sigma_{0}-n^{2}\right)} \overline{\mathbb{Q}} .
$$


We now take $\mathbf{f} \in \mathcal{S}_{k}(C, \psi ; \overline{\mathbb{Q}})$ with $C$ of the form $D\left[\mathfrak{b}^{-1}, \mathfrak{b} \mathfrak{c}\right]$ (i.e. the standard setting in this paper). We are interested in special values of $L(s, \mathbf{f}, \chi)$ for a Hecke character $\chi$ of $F$ of conductor $\mathfrak{f}$.

Theorem 6.2 Let $\mathbf{f} \in \mathcal{S}_{k}(C, \psi ; \overline{\mathbb{Q}})$ be an eigenform for all Hecke operators. Let $\chi$ be a character of $F$ such that $\chi_{\infty}(x)=x_{\infty}^{t}\left|x_{\infty}\right|^{-t}$ with $t \in \mathbb{Z}^{d}$. Define $t^{\prime} \in \mathbb{Z}^{d}$ by $(\psi \chi)_{\infty}(x)=$ $x_{\infty}^{t^{\prime}}\left|x_{\infty}\right|^{t^{\prime}}$. Set $\mu_{v}:=0$ if $\left[k_{v}\right]-t_{v}^{\prime} \in 2 \mathbb{Z}$ and $\mu_{v}=1$ if $\left[k_{v}\right]-t_{v}^{\prime} \notin 2 \mathbb{Z}$. Assume that $m_{0}>$ $[3 n / 2+1]+2$ and either

(1) there exists $v, v^{\prime} \in \infty$ such that $k_{v} \neq k_{v^{\prime}}$, or

(2) $\mu \neq 0$.

Let $\sigma_{0} \in \frac{1}{2} \mathbb{Z}$ be such that

(1) $2 n+1-k_{v}+\mu_{v} \leq \sigma_{0} \leq k_{v}-\mu_{v}$ for all $v \in \infty$,

(2) $\left|\sigma_{0}-n-\frac{1}{2}\right|+n+\frac{1}{2}-\bar{k}+\mu \in 2 \mathbb{Z}^{d}$.

(3) if $n$ is odd, and $\sigma_{0}=n / 2+i$ for $i=0, \ldots n / 2, i \in \mathbb{N}$ or if $n$ is even and $\sigma_{0}=\frac{n-1}{2}+i$, $i=1, \ldots,(n+1) / 2$, then we assume that in Theorem 4.2 we have that $\Lambda_{\mathfrak{c}}(s) / \Lambda_{\mathfrak{h}}(s)=$ 1.

We exclude the cases

(1) $\sigma_{0}=n+\frac{3}{2}, F=\mathbb{Q}, \chi^{2}=1$, and $[k]-t \in 2 \mathbb{Z}$,

(2) $\sigma_{0}=\frac{n}{2}, \mathfrak{c}=\mathcal{O}, n$ is odd and there is no $(\tau, r)$ that satisfy our assumption such that $\rho_{\tau} \neq 1$ and $\chi \psi=1$,

(3) $n / 2<\sigma_{0} \leq n, \mathfrak{c}=\mathcal{O}$ and $(\psi \chi)^{2}=1$.

Let $W$ be a number field so that $\mathbf{f}, \mathbf{f}^{\rho} \in \mathcal{S}_{k}(W)$ and $L \Phi \subset W$, where $\Phi$ is the Galois closure of $F$ in $\overline{\mathbb{Q}}$, and $L$ is as in Theorem 5.2.

If we write $\mathcal{W}:=W(\chi \psi)$ for the extension of $W$ obtained by adjoining the values of the character $\chi \psi$ then,

$$
\frac{L\left(\sigma_{0}, \mathbf{f}, \chi\right)}{\pi^{\beta} D_{F}^{n(n+1) / 4}{ }_{i}^{m} \omega(\overline{\chi \psi})^{\rho}<\mathbf{f}, \mathbf{f}>} \in \mathcal{W},
$$

where $\beta=\pi^{\left.n\left(\sum_{v} k_{v}\right)+d\left(n \sigma_{0}-n^{2}\right)\right)}$, and $m=d$ if [n/2] is odd and 0 otherwise, and $\omega(\cdot)$ is defined by using the Theorem 3.2 as follows

(1) for $\sigma_{0}>n$ and $n$ odd, $\omega(\cdot)$ is as in Theorem $3.2(i)$,

(2) for $\sigma_{0}>n$ and $n$ even, $\omega(\cdot)$ is as in Theorem 3.2 (iii) (a),

(3) for $\sigma_{0} \leq n$ and $n$ odd, $\omega(\cdot)$ is as in Theorem 3.2 (ii),

(4) for $\sigma_{0} \leq n$ and $n$ even, $\omega(\cdot)$ is as in Theorem 3.2 (iv).

Remark 6.3 We refer to [1, p. 171] for explicit conditions so that $\Lambda_{\mathfrak{c}}(s) / \Lambda_{\mathfrak{h}}(s)=1$, in Theorem 4.2.

Proof The proof of this theorem is very similar to Theorem 11 in [1]. We will be applying 4.2 for a proper choice of $(\tau, r)$. The Gamma factors that appear in Theorem 4.2 force us to take $\sigma_{0}>2 n-k_{v}+\mu_{v}$ for all $v \in \infty$, which is the lower bound appearing in the theorem. With $v:=\sigma_{0}-\frac{n}{2}, \beta \in \mathbb{N}$ so that $\pi^{-\beta} D\left(\frac{v}{2}\right) \in \mathcal{N}_{k-l}\left(\Phi \mathbb{Q}_{a b}\right)$, and $\gamma$ as in Theorem 5.2 we obtain for some $\alpha \in \mathbb{Q}^{\times}$,

$$
\begin{aligned}
& \pi^{\gamma} L\left(\sigma_{0}, \mathbf{f}, \chi\right) \psi_{\mathfrak{c}}(\operatorname{det}(r)) c(\tau, r ; \mathbf{f})=D_{F}^{-n(n+1) / 4} \operatorname{det}(\tau)^{s_{0}^{\prime} u+\frac{k+\mu+\frac{n}{2} u}{2}}|\operatorname{det}(r)|_{F}^{n+1-\sigma_{0}} \\
& \quad \times \alpha \prod_{v \in \mathbf{b}} g_{v}\left(\left(\psi / \psi_{\mathbf{c}}\right)\left(\pi_{v}\right) \chi\left(\pi_{v}\right)\left|\pi_{v}\right|^{\sigma_{0}}\right)\left(\Lambda_{\mathfrak{c}} / \Lambda_{\mathfrak{h}}\right)\left(\left(2 \sigma_{0}-n\right) / 4\right)<f, \theta_{\chi}\left(\pi^{-\beta} D(v / 2)\right)>.
\end{aligned}
$$


As in [1], conditions (i) and (ii) are imposed so that the Eisenstein series $D\left(\frac{v}{2}\right)$ of weight $k-\mu-\frac{n}{2}$ for $v=\sigma_{0}-\frac{n}{2}$ are nearly holomorphic (see Theorem 3.1), and (iii) is imposed so that $\left.\frac{\Lambda_{\mathfrak{c}}}{\Lambda_{\mathfrak{h}}}\left(2 \sigma_{0}-n\right) / 4\right)$ does not contribute a pole. Finally the various exclusion follows from various cases where the Eisenstein series $D\left(\frac{\nu}{2}\right)$ is not nearly holomorphic.

We now note that $\theta_{\chi} \in \mathcal{M}_{l}(\mathcal{W})$ and $\pi^{-\beta} D(\nu / 2) \in \mathcal{N}_{k-l}^{r}\left(W \mathbb{Q}_{a b}\right)$ where $r=(k-l-v u) / 2$ if $v>(n+1) / 2$ and $r=(k-l-(n+1-v) u) / 2$ otherwise.

Now we set $P:=D_{F}^{n(n+1) / 4}{ }^{m} \omega(\epsilon \overline{\chi \psi})$ where $\omega(\cdot)$ is defined as in the statement of the theorem. Then by Theorem 3.2 we have that

$$
\operatorname{det}(\tau)^{s_{0}^{\prime} u+\frac{k+\mu+\frac{n}{2} u}{2}} \pi^{-\beta} P^{-1} D(v / 2) \in \mathcal{N}_{k-l}^{r}(\mathcal{W}) .
$$

Indeed, since $L$ is defined to be an extension of $\Phi$ obtained by adjoining $\sqrt{\operatorname{det}(\tau)}$ so that $\mathbf{g}_{\tau, r^{\prime}}$ (with the notation of Theorem 5.2), form a basis, we can pick our $\tau$ so that $c(\tau, r) \neq 0$ from the ones in the basis consisting by $\mathbf{g}_{\tau, r^{\prime}}$. As it is shown in Shimura in [10, Theorem 20.9], after finding a pair $(\tau, q)$ so that $c(\tau, q) \neq 0$ we can modify $q$ to get the pair $(\tau, r)$ with the desired properties. We set $a:=\operatorname{det}(\tau)^{s_{0}^{\prime} u+\frac{k+\mu+\frac{n}{2} u}{2}} \pi^{-\beta} P^{-1}$. In case where $\mu \neq 0$ we have that $\theta_{\chi} D(v / 2) \in \mathcal{R}_{k}$, the space of cuspidal nearly holomorphic modular, and then we can use the operator $\mathfrak{p}: \mathcal{R}_{k} \rightarrow \mathcal{S}_{k}$ of [10, Proposition 15.6] to conclude that there exists $q \in \mathcal{S}_{k}(\mathcal{W})$ so that $\left\langle f, \theta_{\chi} a D(v / 2)>=\left\langle f, q>\right.\right.$. Then by Theorem 5.2 we have that $\frac{\langle f, q\rangle}{\langle f, f\rangle} \in \mathcal{W}$. In the other case, that is of $k$ not being a parallel weight we can use [10, Lemma 15.8], to conclude that there exists a $q \in \mathcal{M}_{k}(W)$ as before. Moreover $k$ not being parallel implies that $\mathcal{M}_{k}=\mathcal{S}_{k}$ (see for example [9, Proposition 10.6 (3)]). Hence we can use Theorem 5.2 to conclude the proof.

We now obtain also some results towards reciprocity laws.

Theorem 6.4 With notation and assumptions as above we have that $A\left(\sigma_{0}, \mathbf{f}, \chi\right)^{\sigma}=$ $A\left(\sigma_{0}, \mathbf{f}^{\sigma}, \chi^{\sigma}\right)$ for all $\sigma \in \operatorname{Gal}(\overline{\mathbb{Q}} / L \Phi)$, where

$$
A\left(\sigma_{0}, \mathbf{f}, \chi\right):=\frac{L\left(\sigma_{0}, \mathbf{f}, \chi\right)}{\pi^{n\left(\sum_{v} k_{v}\right)+d e} D_{F}^{n(n+1) / 4} i^{m} \omega(\overline{\chi \psi})^{\rho} \Omega_{\mathbf{f}}} \in \overline{\mathbb{Q}} .
$$

Proof The proof is similar to Theorem 12 in [1]. We first consider the case where $\mu \neq 0$. Then we have that $\theta_{\chi} \in \mathcal{S}_{l}$. We recall that for $\sigma \in \operatorname{Gal}(\overline{\mathbb{Q}} / \Phi)$ we write $\sigma^{\prime}=\rho \sigma \rho$. Then $\theta_{\chi}^{\sigma^{\prime}}=\theta_{\chi}$, as it follows from the explicit Fourier expansion of $\theta_{\chi}$ (see [1] for details). Moreover arguing as in the theorem above and using the reciprocity laws for Eisenstein series in Theorem 3.2 we have that

$$
\begin{aligned}
& \left(\frac{\pi^{-\beta} D_{F}^{n(n+1) / 4} \operatorname{det}(\tau)^{s_{0}^{\prime} u+\frac{k+\mu+\frac{n}{2} u}{2}} D\left(v / 2, \overline{\psi \chi} \rho_{\tau}\right)}{\omega(\overline{\psi \chi})}\right)^{\sigma^{\prime}} \\
& =\frac{\pi^{-\beta} D_{F}^{n(n+1) / 4} \operatorname{det}(\tau)^{s_{0}^{\prime} u+\frac{k+\mu+\frac{n}{2} u}{2}} D\left(\nu / 2, \overline{\psi^{\sigma} \chi^{\sigma}}\right)}{\omega\left(\epsilon \overline{\psi^{\sigma} \chi^{\sigma}}\right)}, \quad \sigma \in \operatorname{Gal}(\overline{\mathbb{Q}} / L \Phi),
\end{aligned}
$$

Since $\theta_{\chi} D\left(v / 2, \epsilon \overline{\psi^{\sigma} \chi^{\sigma}} \rho_{\tau}\right) \in \mathcal{R}_{k}$, setting $q:=\mathfrak{p}\left(\theta_{\chi} D\left(v / 2, \epsilon \overline{\psi^{\sigma} \chi^{\sigma}} \rho_{\tau}\right)\right) \in \mathcal{S}_{k}$, we have $<f, \theta_{\chi} D\left(\nu / 2, \epsilon \overline{\psi \chi} \rho_{\tau}\right)>=<f, q>$ and $q^{\sigma}=\mathfrak{p}\left(\theta_{\chi}^{\sigma} D\left(\nu / 2, \epsilon \overline{\psi \chi} \rho_{\tau}\right)^{\sigma}\right)$ for all $\sigma \in$ $\operatorname{Gal}(\overline{\mathbb{Q}} / L \Phi)$. In particular, by Theorem 5.2 we have that 


$$
\begin{aligned}
& \left(\frac{D_{F}^{n(n+1) / 4} \operatorname{det}(\tau)^{s_{0}^{\prime} u+\frac{k+\mu+\frac{n}{2} u}{2}}<f, \theta_{\chi} \pi^{-\beta} D\left(\nu / 2, \overline{\psi \chi} \rho_{\tau}\right)>}{\omega(\overline{\psi \chi})^{\rho} \Omega_{\mathbf{f}}}\right)^{\sigma} \\
& =\frac{D_{F}^{n(n+1) / 4} \operatorname{det}(\tau)^{s_{0}^{\prime} u+\frac{k+\mu+\frac{n}{2} u}{2}}<f^{\sigma}, \theta_{\chi^{\sigma}} \pi^{-\beta} D\left(\nu / 2, \overline{\psi^{\sigma} \chi^{\sigma}} \rho_{\tau}\right)>}{\omega\left(\overline{\psi^{\sigma} \chi^{\sigma}}\right)^{\rho} \Omega_{\mathbf{f}^{\sigma}}},
\end{aligned}
$$

from which we conclude the proof of the theorem. For the other case we use, as in the previous theorem, the result of Shimura [10, Lemma 15.8] to conclude theta there exist $q \in \mathcal{M}_{k}(W)=\mathcal{S}_{k}(W)$ so that

$$
\frac{D_{F}^{n(n+1) / 4} \operatorname{det}(\tau)^{s_{0}^{\prime} u+\frac{k+\mu+\frac{n}{2} u}{2}}<f, \theta_{\chi} \pi^{-\beta} D\left(v / 2, \overline{\psi \chi} \rho_{\tau}\right)>}{\omega(\overline{\psi \chi})^{\rho} \Omega_{\mathbf{f}}}=\frac{<f, q>}{\Omega_{\mathbf{f}}} .
$$

For the reciprocity we use Proposition 14.13 in [10] to conclude that the above equation is Galois equivariant. Indeed the result of Shimura gives that the space of nearly holomorphic Siegel modular forms has an $\Phi$ rational basis and it is finite dimensional thanks to [10, Lemma 14.3]. So the map of Lemma 15.8 of [10] is equivariant over $\Phi$.

We conlcude this paper by making a few remarks. In [1] we were able, thanks to a result of Harris [5] on the rationality of Eisenstein series, to establish results without $\mu \neq 0$ or nonparallel weight. Of course we had to assume that the critical value is in the range of absolute convergence, since only then one can employ the result of Harris. However in our situation the results of Harris in [5] cannot be applied since half-integral weight modular forms cannot be understood using Shimura varieties. In our forthcoming work [3] we address this questions and obtain results in this direction. We also mention a recent work of Mercuri [6], where he obtains results in the case of $\mu=0$ (in the notation of Theorem 6.2 above) when $F=\mathbb{Q}$ and $n$ is odd.

We mention that there is yet another integral expression of the $L$-values of half-integral Siegel modular forms, namely the doubling method (see [10]). The two methods are not equivalent and we refer to the last section of [1], where the integral weight case is discussed, for a discussion of how the two methods compare.

Open Access This article is distributed under the terms of the Creative Commons Attribution 4.0 International License (http://creativecommons.org/licenses/by/4.0/), which permits unrestricted use, distribution, and reproduction in any medium, provided you give appropriate credit to the original author(s) and the source, provide a link to the Creative Commons license, and indicate if changes were made.

\section{References}

1. Bouganis, Th: On special L-values attached to Siegel modular forms. In: Bouganis, Th, Venjakob, O. (eds.) Iwasawa Theory 2012 - State of the Art and Recent Advances, Contributions in Mathematical and Computational Sciences, vol. 7, pp. 135-176. Springer, Berlin, Heidelberg (2014)

2. Bouganis, Th: On the algebraicity of special L-values of Hermitian modular forms. Doc. Math. 20, $1293-1329(2015)$

3. Bouganis, Th.: On the rationality of Eisenstein Series of symplectic and unitary groups (in preparation)

4. Feit, P.: Poles and residues of Eisenstein series for symplectic and unitary groups. Mem. Am. Math. Soc. 346, 61 (1986)

5. Harris, M.: Eisenstein series on Shimura varieties. Ann. Math. 119, 59-94 (1984)

6. Mercuri, S.: Special values of zeta functions for modular forms of half-integral weight (preprint)

7. Shimura, G.: Zeta functions and Eisenstein series on metaplectic groups. Invent. Math. 121, 21-60 (1995) 
8. Shimura, G.: Convergence of zeta functions on symplectic and metaplectic groups. Duke Math. J. 82(2), 327-347 (1996)

9. Shimura, G.: Euler products and Eisenstein series. CBMS Reg. Conf. Seri. Math. 93 (1997)

10. Shimura, G.: Arithmeticity in the theory of automorphic forms. Math. Surv. Monogr. 82 (2000)

11. Sturm, J.: The critical values of zeta functions associated to the symplectic group. Duke Math. J. 48(2), 327 (1981) 